\title{
Platelet-Rich Plasma in Reconstruction of Posterior Meatal Wall after Canal Wall Down Mastoidectomy
}

\author{
Mohammad El-Sayed Abd Elbary ${ }^{1}$ Wail Fayez Nasr ${ }^{2}$ Samir Sorour Sorour ${ }^{2}$ \\ ${ }^{1}$ Department of Otorhinolaryngology and Head and Neck Surgery, \\ Faculty of Medicine, Zagazig University, Zagazig, Egypt \\ 2 Department of Otorhinolaryngology and Head and Neck Surgery, \\ Faculty of Human Medicine, Zagazig University, Zagazig, \\ El Sharkya, Egypt

\begin{abstract}
Address for correspondence Wail Fayez Nasr, MD, Department of Otorhinolaryngology and Head and Neck Surgery, Faculty of Human Medicine, Zagazig University, 12 Abo Bakr Esedeek Villat El Gamma Zagazig, Zagazig, El Sharkya 4415, Egypt

(e-mail: wail.fayez@yahoo.com).
\end{abstract}

Int Arch Otorhinolaryngol 2018;22:103-107.

\begin{abstract}
Introduction Canal wall down (CWD) mastoidectomy has many drawbacks, including chronic otorrhea not responding to medications, granulations, dizziness on exposure to cold or hot water, and tendency of debris accumulation in the mastoid cavity, demanding periodic cleaning. Many of these problems can be solved by reconstruction of the posterior meatal wall (PMW).

Objectives To assess the results of PMW reconstruction after CWD mastoidectomy for cholesteatoma using titanium mesh and platelet-rich plasma (PRP) mixed with bone pate. Methods This study was conducted with 20 patients that have atticoantral chronic suppurative otitis media. All cases were subjected to CWD mastoid surgery with complete elimination of the disease and reconstruction of the PMW by titanium mesh and the mixture of PRP with bone pate. All patients were exposed to a full preoperative evaluation and full postoperative assessment of the complications, the appearance of the external auditory canal contour, and the hearing gain expressed by the change of the air bone gap postoperatively.

Results The PMW reconstructed by titanium mesh and the mixture of PRP with bone pate showed a smooth contour. During the follow-up of 12 to 36 months, the postoperative appearance of the external auditory canal contour was found to be smooth without hidden pouches, irregularities or stenosis in all cases. No granulation, foreign body reaction, or extrusion and/or displacement of the titanium mesh were

\section{Keywords}

- platelet-rich plasma

- titanium

- cholesteatoma registered. No facial palsy or recurrent cholesteatoma was reported.

Conclusion The surgical reconstruction of the PMW using PRP, bone pate and titanium mesh after CWD mastoidectomy appears to be reliable without considerable complications, giving a smooth appearance to the PMW.
\end{abstract}

\section{Introduction}

The goals of the surgery for cholesteatoma are to eradicate the disease and prevent its recurrence. Canal wall up (CWU) mastoidectomy preserves the posterior canal wall, obviating the need for repeated mastoid cavity cleaning and preventing recurrent mastoid cavity infections. However, the recurrence rate is very high $(\sim 36 \%)$ in adults and in children $(67 \%){ }^{1}$

In the canal wall down (CWD) mastoidectomy, the posterior meatal wall (PMW) is removed, providing exposure of the received

November 11, 2016

accepted

March 9, 2017

published online

May 17, 2017
DOI https://doi.org/

10.1055/s-0037-1602694. ISSN $1809-9777$.
Copyright $(2018$ by Thieme Revinter

Publicações Ltda, Rio de Janeiro, Brazil
License terms

(ㄷ) (i) $\ominus$ (\$) 
entire attic and middle ear, helping to ensure complete disease eradication. Hence, the recurrence rate is as low as $2 \%$ for this procedure. ${ }^{2-4}$ However, CWD mastoidectomy has many drawbacks, such as cavity problems (chronic otorrhea, granulations, dizziness in cold or hot water, and accumulation of debris in the exteriorized mastoid cavity, requiring periodic cleaning), social handicaps (non-esthetic meatoplasty, water restrictions to prevent bowl infections) and/ or hearing problems (major conductive or mixed hearing loss as the ossicular chain reconstruction is very difficult, as is the insertion of a hearing aid). 5,6

CWD mastoidectomy followed by concomitant reconstruction of the PMW achieves the low recurrence rate of cholesteatoma after it besides the advantages of CWU mastoidectomy. This provides a structural support, which plays an important role in the prevention of the postoperative formation of a retraction pocket and subsequent cholesteatoma development. ${ }^{6-8}$

The materials used for PMW reconstruction can be classified into two main categories: autologous grafts (bone, cartilage) and synthetic materials. ${ }^{9}$

These grafts have limited supply, ${ }^{10}$ and can be associated with donor-site morbidity and new cavity formation after tissue retraction. ${ }^{11}$ Many of these problems can be avoided with the synthetic materials, ${ }^{12}$ which should be available, non-expensive, and biocompatible, and should not be extruded or resorbed, ${ }^{13}$ such as titanium mesh. ${ }^{12}$

Titanium is biocompatible and can osteointegrate with the bone; ${ }^{14}$ in addition, titanium mesh is a malleable material, so it can be easily shaped according to the surgical requirements. ${ }^{15}$

Platelet-rich plasma (PRP) is an autologous product derived from whole blood through the process of gradient density centrifugation. Autologous PRP has been shown to be safe and effective in promoting the natural processes of wound healing, soft tissue reconstruction, bone reconstruction and augmentation. ${ }^{16}$

The aim of this study was to assess the results of PMW reconstruction after CWD mastoidectomy for cholesteatoma using titanium mesh and PRP mixed with bone pate intraoperatively.

\section{Patients and Methods}

This study was conducted from May 2012 to February 2015 on 20 patients who had cholesteatoma; there were 9 males and 11 females, with ages ranging from 12 to 36 years. These patients underwent CWD mastoidectomy followed by concomitant reconstruction of the PMW using titanium mesh and PRP mixed with bone pate in the Otolaryngology Head and Neck Surgery Department of our institution. All patients had atticoantral chronic suppurative otitis media (cholesteatoma).

Revision cases, patients with intracranial complications and labyrinthine fistula were excluded. An informed consent form was signed by every patient included in this study, and approval was obtained from the institution's Review Board.

All patients were subjected to history-taking, and thorough general and otorhinological examinations, including a microscopic ear examination. Pure tone audiometry (PTA) was performed preoperatively and one year postoperatively. The air conduction threshold for each patient was calculated as the mean of the $0.5,1,2$, and $4 \mathrm{kHz}$ thresholds. ${ }^{17}$ Computed tomography (CT) scans of the temporal bone were performed in all patients both preoperatively and one year postoperatively.

\section{Surgical Techniques}

Under general anesthesia, during anesthesia induction, the PRP was prepared by taking $5 \mathrm{cc}$ of autologous blood from the patient and processing it during the mastoidectomy using a laboratory centrifuge (Heraeus labofuge 200, Germany) (-Fig. 1). Then, CWD mastoidectomy was started by postauricular incision, and the postauricular superiorly based mucoperiosteal flap was created. The large temporalis fascia graft was then prepared. Canal wall down mastoidectomy with lowering of the facial ridge was performed, but earlier in this step, a sufficient amount of bone pate was collected from the healthy bone of the mastoid, the squamous temporal bone, and the zygomatic root. The PRP was mixed with the obtained bone pate to form a paste.

\section{Reconstruction of the Posterior Meatal Wall}

The titanium mesh used in this study was $0.1 \mathrm{~mm}$ thick, with $2 \mathrm{~mm}$ pores (Titanium Micromesh, JEIL medical corporation, Seoul, Korea). Two deep grooves in the bone along the facial ridge posteriorly and inferiorly and along the anterior-superior wall of the meatus were drilled with a 2 to $3 \mathrm{~mm}$ cutting burr. A foil template gave the accurate measurements, and the curvature of the posterior canal wall was used to get the template of the PMW. Using the foil template as a model, the prosthetic wall was cut from the titanium mesh, and a little piece from the mesh like a tail was extended from the prosthetic wall. The mesh was then folded and placed into the created grooves, and the tail extended from the mesh was fixed with one titanium screw to the cavity border to allow for a better fixation of the prosthetic wall. The PRP and bone pate paste was used to cover the meatal surface of the mesh completely. The titanium mesh supports the layer of PRP bone pate paste that will become viable bone later on. (-Fig. 2)

The prepared temporalis fascia graft was used to reconstruct the tympanic membrane perforation and extended over the newly reconstructed PMW. (-Fig. 3)

Thereafter, the prepared post-auricular superiorly based mucoperiosteal flap was rotated into the mastoid cavity, obliterating it and supporting the newly reconstructed PMW. Then, the tympanomeatal flap was repositioned over the newly reconstructed PMW, and was secured in its position using Gelfoam (Pfizer, New York, NY, US).

Therefore, the newly reconstructed PMW consisted of the titanium micromesh, the PRP and bone pate sheet, the temporalis fascia graft and the tympanomeatal flap. Lastly, the post-auricular incision was closed in layers.

The temporalis fascia graft was applied over the newly reconstructed PMW before repositioning of the tympanomeatal flap to avoid any possibility of exposure of any part of 


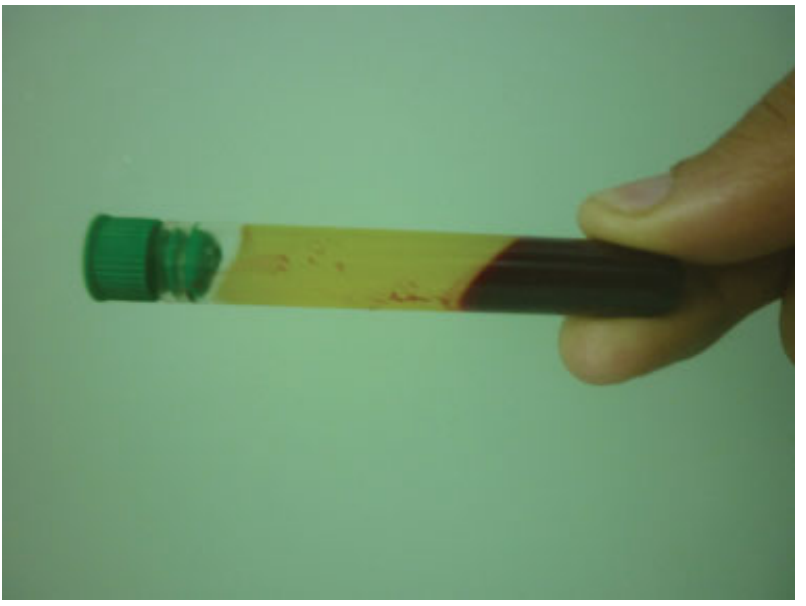

Fig. 1 Platelet-rich plasma.

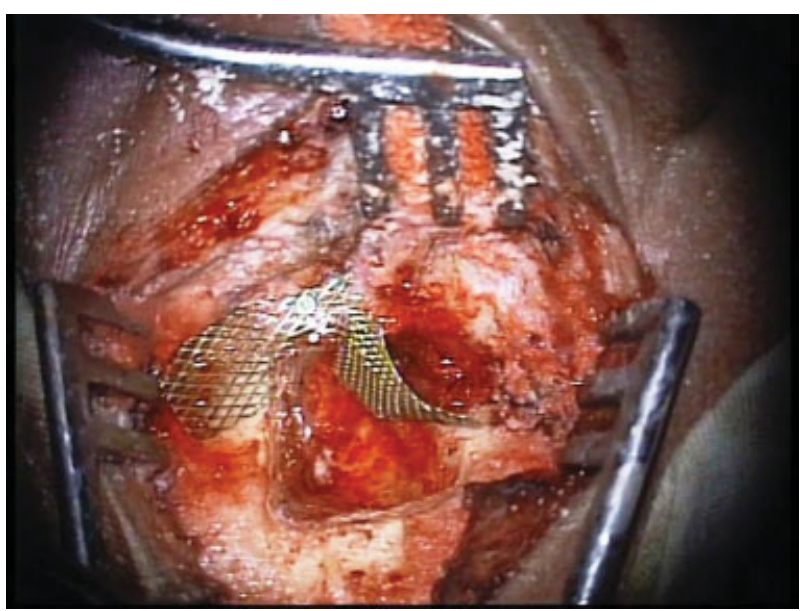

Fig. 2 Titanium mesh inserted between the two grooves with fixation of the titanium tail by titanium screw.

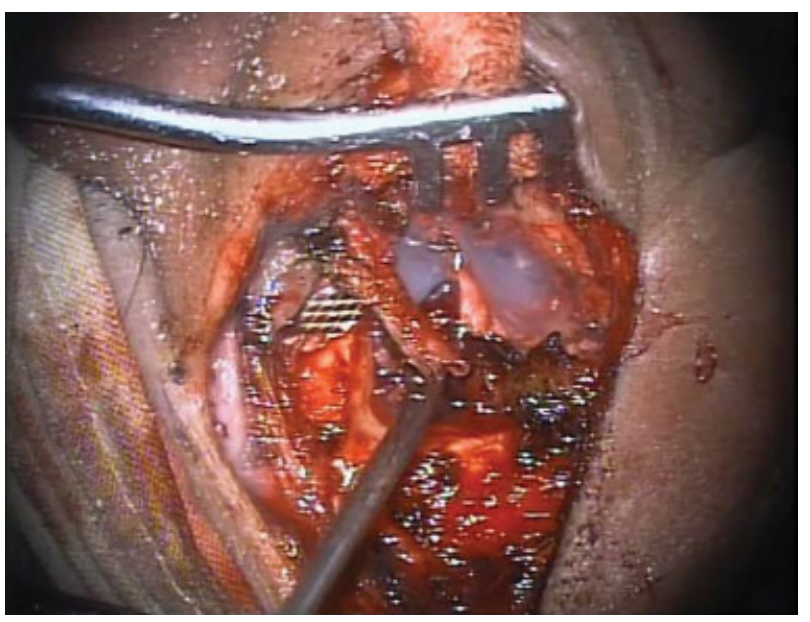

Fig. 3 Posterior meatal wall completely reconstructed by titanium mesh and PRP and bone pate paste, and the tympanic membrane reconstructed by temporalis fascia.

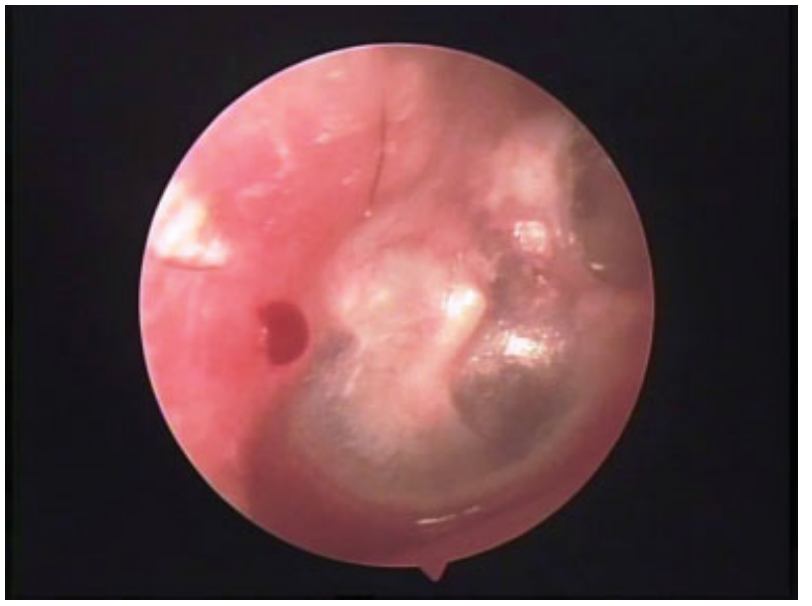

Fig. 4 Follow-up of a case operated 12 months before.

the new wall, as the flap is not always kept intact during the elevation.

The dressing and the stitches were removed one week postoperatively. Otoscopic and otoendoscopic examinations were performed weekly for the first month, then monthly for 3 months, then every 3 months afterwards (-Fig. 4). Pure tone audiometry and a CT of the temporal bone were taken one year postoperatively (-Fig. 5). The postoperative hearing gain was calculated as the preoperative air conduction threshold minus the postoperative one; the bone conduction was not evaluated, as we had used the change of air bone gap as a parameter for the evaluation, and all patients included in the study were having pure conductive hearing loss preoperatively, and there were no cases in which the bone conduction was changed postoperatively.

\section{Statistical Analysis}

Statistical analysis was conducted using the Statistical Package for the Social Sciences (SPSS Inc, Chicago, IL, US) statistical software, version 14.0 for Windows. Values of $p<0.05$ were considered statistically significant.

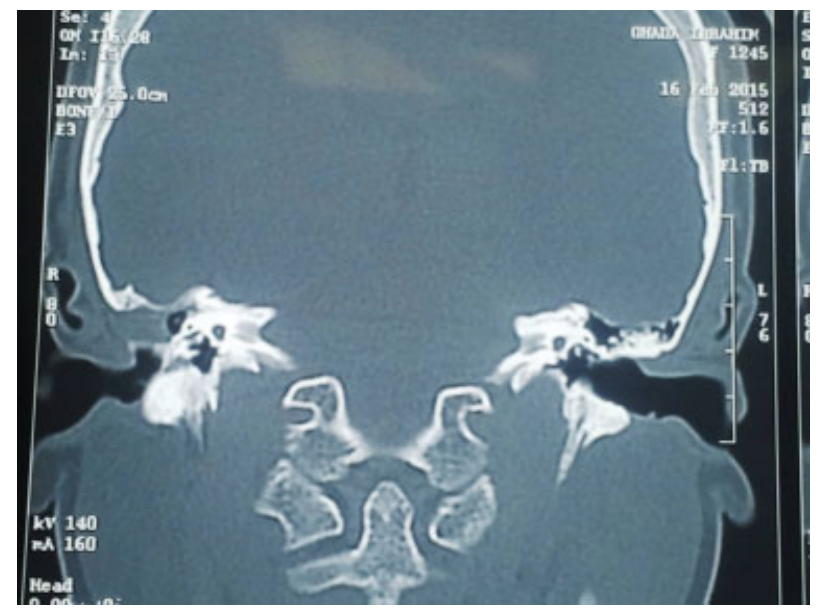

Fig. 5 Coronal CT showing the reconstructed PMW after 12 months. 


\section{Results}

This study included 20 patients who had cholesteatoma; 9 males and 11 females, with ages ranging from 12 to 36 years (mean $23.1 \pm 8.4$ ).

No difficulty was reported during the fashioning and the building of the new PMW by titanium mesh and PRP and bone pate paste.

During the follow-up of 12 to 36 months (the mean followup was $24.3 \pm 7.49$ months), the postoperative appearance of the external auditory canal contour was smooth without hidden pouches, irregularities or stenosis in all cases, and no granulation, foreign body reaction, extrusion and/or displacement of the titanium mesh (that means that there was good osteointegration of the titanium mesh) were registered. No facial palsy or recurrent cholesteatoma was reported. This was evaluated not only by clinical examination, but also by a CT that was performed in all cases one year postoperatively

Postoperative temporary otorrhea had occurred in 2 ears (10\%), and it stopped with antibiotics (for 7 days) and strict water precautions.

The preoperative air bone gab (ABG) ranged from 20 to $42 \mathrm{~dB}$, with a mean of $28.1 \pm 6.3 \mathrm{~dB}$. The postoperative $\mathrm{ABG}$ ranged from 22 to $42 \mathrm{~dB}$, with a mean of $29.6 \pm 6.1 \mathrm{~dB}$ (-Table 1).

\section{Discussion}

In CWU mastoidectomy, the PMW is preserved avoiding the need for recurrent bowl cleansing and preventing recurrent bowl infection. But the recurrence rate is high ( $\sim 36 \%$ in adults and $67 \%$ in children). ${ }^{1}$ On the other hand, CWD mastoidectomy is an easier operation, requires less operative time, and calls for less surgical experience than the CWU procedures, with lower recurrence and residual rate, so it is the most commonly used surgical procedure for the management of cholesteatoma worldwide. ${ }^{17}$

The surgical techniques used for the management of open mastoid cavity after CWD mastoidectomy include obliteration (cavity filling), reconstruction (canal wall defect repair) and ablation (external canal closure). The obliteration technique involves filling the mastoid cavity with pedicled flaps and/or various materials, such as fat, bone chips and hydroxyapatites. The ablation or canal closure technique is only recommended in certain cases, such as a deaf ear with severe otorrhea. ${ }^{18}$

We have used a combination of both PMW reconstructions in addition to mastoid cavity obliteration. We used the titanium mesh and PRP and bone pate paste in PMW reconstruction that was tailored and built-up during surgery. Then, we managed to hold the newly reconstructed PMW from the mastoid surface through mastoid obliteration by a superiorly based mucoperiosteal flap.

Titanium has been widely used in maxillofacial, otolaryngological and surgical reconstructive procedures. ${ }^{19-21}$ It has been proven to be biocompatible and osteointegrative. ${ }^{14}$ Platelet-rich plasma has been shown to be safe and effective in promoting the natural processes of wound healing, soft tissue reconstruction, and bone reconstruction and augmentation. ${ }^{16}$

In this procedure, the PMW was reconstructed by titanium mesh and PRP and bone pate paste, while in the study conducted by Zini et al study, the PMW was reconstructed by titanium mesh, but the bone pate was mixed with fibrin glue. ${ }^{15}$ Platelet-rich plasma differs from fibrin glue, as PRP has a high concentration of platelets and normal concentration of fibrinogen, whereas autologous fibrin glue can be created from platelet-poor plasma and consist primarily of fibrinogen (commercial fibrin glues are created from pooled homologous human donors). The high concentration of platelets in PRP promotes wound healing, bone growth, and tissue sealing. ${ }^{16}$

Platelets have been shown to stimulate the mitogenic activity of human trabecular bone cells and to increase the proliferation rate of human osteoblast-like cells and stromal stem cells, thus contributing to the regeneration of mineralized tissues. Growth factors released from platelets signal local mesenchymal and epithelial cells to migrate, divide, and increase the synthesis of collagen and matrix, thus providing a scaffold that encourages the migration of osteoblasts. Growth factors contained in PRP also stimulate chemotaxis, metabolism, and proliferation in osteoblasts and in bone marrow osteoprogenitor cells. ${ }^{22,23}$

In a study conducted by Sudhoff et al, the PMW was reconstructed with a composite cartilage titanium mesh. ${ }^{24}$ In this study, the mesh surface must be completely covered with cartilage, so a large amount of cartilage should be harvested from the concha and fixed to the mesh by absorbable sutures, which is time-consuming with donor site morbidity, while in our study the PRP and bone pate paste is easily prepared and less time-consuming.

With regards to the stability, the newly reconstructed PMW by titanium mesh and PRP and bone pate paste was stable when applied, with no reported extrusion or displacement. This agrees with Zini et $\mathrm{al}^{15}$ and Sudhoff et al. ${ }^{24}$

Silicone, proplast, ionomer cement, ceravital and hydroxyl apatite are examples of synthetic materials that have been

Table 1 Preoperative and postoperative air bone gab (ABG)

\begin{tabular}{|l|c|c|c|c|}
\hline Air bone gab (ABG) & $\begin{array}{c}\text { Preoperative } \\
(\boldsymbol{N}=\mathbf{2 0})\end{array}$ & $\begin{array}{c}\text { Postoperative } \\
(\boldsymbol{N}=\mathbf{2 0})\end{array}$ & WSR & $\begin{array}{c}p \text {-value } \\
(\text { Sig })\end{array}$ \\
\hline Mean \pm SD & $28.1 \pm 6.3$ & $29.6 \pm 6.1$ & \multirow{2}{*}{-1.250} & 0.211 \\
Median & 27.5 & 27.5 & & \\
\cline { 1 - 3 } Range & $20-42$ & $22-42$ & & \\
\hline
\end{tabular}

Abbreviation: SD, standard deviation; Sig, significance; NS, non significant; WSR, Wilcoxon Signed Ranks test.

Note: Values of $p<0.05$ were considered statistically significant. 
used in PMW reconstruction. But silicone leads to foreign body reaction; ${ }^{25}$ the use of proplast leads to dehiscence problems; ${ }^{26}$ ionomer cement leads to infection and encephalopathy; ${ }^{27}$ and ceravital leads to absorption and lysis. ${ }^{13,28}$

In this study, the PRP and bone pate paste proved to be easily prepared and applied over the titanium mesh. Postoperative infection had occurred only in 2 cases (10\%), which responded well to antibiotics and conservative treatment, and this confirms the wound healing promotion effect of the PRP. ${ }^{16}$

Sudhoff et al $^{24}$ registered the occurrence of granulations in 2 patients, apparently due to incomplete coverage of the titanium mesh by cartilage. Zini et $\mathrm{al}^{15}$ reported the development of granulations in one patient. In our study, no granulations were detected, and this may be explained by the healing promotion effect of the PRP, which was mixed with the bone pate, allowing for the complete covering of the mesh surface and encouraging healing and bone formation.

In this study, all patients (100\%) showed smooth and apparently normal PMW contours, which agree with Zini et $\mathrm{al}^{15}$ and Sudhoff et al. ${ }^{24}$

Regarding the postoperative hearing changes in our study, there were no significant changes in hearing in which the mean pure tone average air-bone gap had increased from $28.1 \mathrm{~dB}$ to $29.6 \mathrm{~dB}$ (in this study, ossiculoplasty was not performed, as the goal was to reconstruct the posterior meatal wall mainly, then the ossicles could be reconstructed later on in another set of surgeries), and this agrees with Zini et $\mathrm{al}^{15}$ and Sudhoff et al. ${ }^{24}$

So, PRP appears to be reliable and safe, as it encourages the bone formation of the PMW effectively and improves healing with no reported complications for the surgical reconstruction of the PMW.

\section{Conclusion}

Surgical reconstruction of the PMW using PRP with titanium mesh after CWD mastoidectomy appears to be reliable and devoid of considerable complications, giving a smooth appearance to the PMW and improving healing. But the follow-up period is relatively short, so we recommend further studies with longer follow-up periods to evaluate our results.

\section{References}

1 Shohet JA, de Jong AL. The management of pediatric cholesteatoma. Otolaryngol Clin North Am 2002;35(04):841-851

2 Palva T. Surgical treatment of chronic middle ear disease. II. Canal wall up and canal wall down procedures. Acta Otolaryngol 1987; 104(5-6):487-494

3 Gantz BJ, Wilkinson EP, Hansen MR. Canal wall reconstruction tympanomastoidectomy with mastoid obliteration. Laryngoscope 2005;115(10):1734-1740

4 Babighian G. Posterior and attic wall osteoplasty: hearing results and recurrence rates in cholesteatoma. Otol Neurotol 2002;23(01):14-17

5 Dornhoffer JL. Surgical modification of the difficult mastoid cavity. Otolaryngol Head Neck Surg 1999;120(03):361-367

6 Gehrking E. Osteoplastic atticoantrotomy with autologous bone chips and a bony attic strut in cholesteatoma surgery. Eur Arch Otorhinolaryngol 2010;267(07):1055-1066
7 Hinohira Y, Yanagihara N, Gyo K. Improvements to staged canal wall up tympanoplasty for middle ear cholesteatoma. Otolaryngol Head Neck Surg 2007;137(06):913-917

8 Kronenberg J, Shapira Y, Migirov L. Mastoidectomy reconstruction of the posterior wall and obliteration (MAPRO): preliminary results. Acta Otolaryngol 2012;132(04):400-403

9 Chan CY, Chan YM. Mastoid Obliteration and Reconstruction: A Review of Techniques and Results. Proceedings of Singapore Healthcare 2012;21(01):23-29

10 Leatherman BD, Dornhoffer JL. The use of demineralized bone matrix for mastoid cavity obliteration. Otol Neurotol 2004; 25(01):22-25, discussion 25-26

11 Peitoia M. Use of Bioactive Glass S53P4 In frontal sinus obliteration and in reconstruction of facial bone defects. Vivox-id report. Summary Clinical Trials 2004;30(04):16-20

12 Della Santina CC, Lee SC. Ceravital reconstruction of canal wall down mastoidectomy: long-term results. Arch Otolaryngol Head Neck Surg 2006;132(06):617-623

13 el-Seifi A, Fouad B. Long-term fate of Plastipore in the middle ear. ORL J Otorhinolaryngol Relat Spec 1998;60(04):198-201

14 van der Pouw CTM, Johansson CB, Mylanus EAM, Albrektsson T, Cremers CW. Removal of titanium implants from the temporal bone: histologic findings. Am J Otol 1998;19(01):46-51

15 Zini C, Quaranta N, Piazza F. Posterior canal wall reconstruction with titanium micro-mesh and bone patè. Laryngoscope 2002; 112(04):753-756

16 Smith RG, Gassmann CJ, Campbell M. Platelet-rich Plasma: Properties and Clinical Applications. The Journal of Lancaster General Hospital 2007;2(02):73-78

17 Hulka GF, McElveen JT Jr. A randomized, blinded study of canal wall up versus canal wall down mastoidectomy determining the differences in viewing middle ear anatomy and pathology. Am J Otol 1998;19(05):574-578

18 Sasaki Y, Iino Y, Suzuki J. Posterior canal wall reconstruction tympanoplasty for operated ears with open mastoid. Acta Otolaryngol 2002;122(03):249-254

19 Jung TTK, Park SK. Reconstruction of mastoidectomy defect with titanium mesh. Acta Otolaryngol 2004;124(04):440-442

20 Nakayama B, Hasegawa Y, Hyodo I, et al. Reconstruction using a three-dimensional orbitozygomatic skeletal model of titanium mesh plate and soft-tissue free flap transfer following total maxillectomy. Plast Reconstr Surg 2004;114(03):631-639

21 Stupp CH, Stupp HF, Grün D. [Replacement of ear ossicles with titanium prostheses]. Laryngorhinootologie 1996;75(06):335-337

22 Lind M. Growth factor stimulation of bone healing. Effects on osteoblasts, osteomies, and implants fixation. Acta Orthop Scand Suppl 1998;283:2-37

23 Marx RE. Platelet-Rich Plasma. A Source of Multiple Autologous Growth Factors for Bone Grafts. In: Lynch SE, Genco RJ, Marx RE, eds. Tissue Engineering: Applications in Maxillofacial Surgery and Peridontics. Chicago: Quintessence Publishing Co, Inc; 1999: 71-82

24 Sudhoff H, Brors D, Al-Lawati A, Gimenez E, Dazert S, Hildmann H. Posterior canal wall reconstruction with a composite cartilage titanium mesh graft in canal wall down tympanoplasty and revision surgery for radical cavities. J Laryngol Otol 2006;120(10):832-836

25 Rosenblut B, Ahlvin RC, Carr CD, Senturia BH. Silicone implants in the mastoid portion of the temproal bone. Ann Otol Rhinol Laryngol 1966;75(03):889-902

26 Shea JJ Jr, Malenbaum BT, Moretz WH Jr. Reconstruction of the posterior canal wall with Proplast. Otolaryngol Head Neck Surg 1984;92(03):329-333

27 Renard JL, Felten D, Béquet D. Post-otoneurosurgery aluminium encephalopathy. Lancet 1994;344(8914):63-64

28 Reck R, Störkel S, Meyer A. Bioactive glass-ceramics in middle ear surgery. An 8-year review. Ann N Y Acad Sci 1988;523:100-106 